\title{
The electron temperature in the plasma of a spark-recombination laser
}

\author{
M. Villagrán, , M. Gallardo, ${ }^{\text {b) }}$ and J. O. Tocho ${ }^{\text {) }}$ \\ Centro de Investigaciones Opticas, Comisión de Investigaciones Científicas de la Provincia de Buenos Aires, \\ 1900 La Plata, Argentina \\ 1. S. Rocca \\ Colorado State University, Fort Collins, Colorado 80523
}

(Received 10 October 1986; accepted for publication 26 November 1986)

The electron temperature in an expanding spark plasma used as active medium in a cadmiumrecombination laser was measured using the relative intensities of helium lines originated from levels in local thermodynamic equilibrium. Measurements resolved in space and time show that the electron temperature at the conditions of maximum gain are 1000 and $600 \mathrm{~K}$ at 5 and $10 \mathrm{~mm}$ of the sparks, respectively. Rapid cooling occurs after the termination of the dischargecurrent pulse, and the electron temperature reaches values below $1000 \mathrm{~K}$. Subsequentily, the electron temperature decrease is slow. The optical gain calculated for the $1.43-\mu \mathrm{m} \mathrm{Cd}$ (I)recombination-laser transition using the measured electron temperature and particle density values is $3 \times 10^{-3} \mathrm{~cm}^{-1}$, in good agreement with measurements.

\section{INTRODUCTION}

The basic principles of the generation of coherent radiation in plasma recombination were developed theoretically in a series of papers by Gudzenko et al ${ }^{1,2}$ Different excitation methods and elements have been used to produce recombining plasmas emitting in several regions of the spectrum..$^{3-7}$ A simple way to produce recombination-laser action using metal vapors was developed by Silfvast et al ${ }^{8}$ The device, called SPER for segmented plasma excitation and recombination, produces segmented plasmas by vaporizing the laser material with high-voltage, high-current pulses applied to the end of a series of thin, narrow, metal strips glued on an insulating material. The metal vapor plasmas expand hemispherically, cool in a buffer gas, and recornbine.

The plasma electron temperature $T_{e}$ plays a very impor. tant role in recombination lasers. In the conventional theory of three-body collisional recombination, the recombination rate that populates the laser upper levels depends on $T_{e}^{--9 / 2}$ (Ref. 9). The laser gain consequently depends strongly on the value of the electron temperature. Also, the de-excitation of the laser upper levels by superelastic electron collisions is dependent on the eiectron temperature. Thereby, the maximum plasma density at which the laser can operate is a function of $T_{e}$. Wood et al. have derived a simple expression to estimate this plasma density as a function of the electron temperature and wavelength of the laser transition. ${ }^{10}$

In the absence of electron temperature measurements in SPER discharges, $T_{e}$ values corresponding to other recombination-laser plasmas have been used in SPER lasers calculations. ${ }^{10}$ An electron temperature of $3000 \mathrm{~K}$, corresponding to the afterglow of an axially excited $\mathrm{Sr}$ (II)-recombination laser, ${ }^{3}$ has been used to calculate rates in SPER discharges. ${ }^{10}$ Optical gains for CdI laser lines calculated using this value

\footnotetext{
" Graduate Student, University of La Plata, supported by CONICET, Argentina.

b) Member of "Carrera del Investigador Cientifico y Tecnológico," CONICET.
}

of $T_{e}$ and measured plasma densities differ from measurements by two orders of magnitude.

We have done measurements of $T_{c}$ in a SPER discharge for the first time that are resolved in time and space. The density of recombining cadmium ions was also measured in the same device to allow for consistency in laser gain calculations.

A SPER device having 33 similar sparks was used to measure the gain in the $1.43-\mu \mathrm{m} \mathrm{Cd}$ (1) laser line. The value of the gain calculated using the measured values of $T_{e}$ at the time and position of maximum amplification is in good agreement with the experimental measurements.

\section{EXPERIMENT}

$\mathrm{Cd}^{+}$density measurements in $\mathrm{Cd}(\mathrm{I})$-recombination lasers have been previously reported. ${ }^{10}$ We repeated the measurements with the purpose of allowing a more valid comparison between the gain calculated using the measured electron temperature and the experimental value. In this way, all the parameters used in the calculation and, the gain itself, are known to be measured using the same sparks and under very similar conditions.

A simple SPER device similar to that designed by Silfvast et al. ${ }^{8}$ was used to produce laser action in cadmium vapor. Thirty-three $1-\mathrm{mm}$-thick by 2 -mm-wide cadmium 10 -mm long strips with a $1-\mathrm{mm}$ gap between them were used. The strips were fixed to a glass plate and installed in a gas cell filled with helium at a pressure of 10 Torr. The buffer gas was circulated at a low rate by use of needle valves in order to remove impurities after the fires. Two internal, long-radii gold mirrors were used to produce osciliations in several infrared lines of $\mathrm{Cd}$ (I). The coupling was made by a $2-\mathrm{mm}$ diam hole in the output mirror. The strips were allowed to be moved parallel to, and from 5 to $15 \mathrm{~mm}$ of the axis of the resonator.

A 20-nF low-inductance capacitor charged up to $10 \mathrm{kV}$ produced 42-A peak-current pulses of 450-ns FWHM when switched by a pressurized spark gap triggered at a $10 \mathrm{~Hz}$ 
repetition rate. The laser oscillation of three lines in the near infrared at $1.398,1.433$, and $1.448 \mu \mathrm{m}$ was detected by a room-temperature Ge diode and a $50-\mathrm{cm}$ focal length monochromator. The 1.433- $\mu \mathrm{m}$ line was the most intense. The laser oscillation starts approximately $25 \mu \mathrm{s}$ after the current pulse and has a typical duration of $30 \mu \mathrm{s}$.

The electronic temperature and the ionic- and atomiccadmium ground-state densities were measured using a reduced three-strip configuration. Only one gap was fired in the temperature measurements. One and two gaps were used to measure the atomic- and ionic-ground-state densities by use of the resonant absorption technique. "An external lowinductance resistor was used in this part of the experiment in order to reproduce the electronic-discharge-current pulse of the 33-strip laser. The light from the reduced device was focused using quartz optics on the sit of an Ebert monochromator. The detection was made by photomultipliers (EMI $9634 \mathrm{QB}$ in the UV and Hamamatsu R446 in the visible) using a Boxcar integrator îriggered by a current-coil monitor.

\section{A. Electronic temperature measurements}

We have measured the relative intensity of the $\mathrm{He}$ (I) lines in the $2{ }^{3} S-n p{ }^{3} P$ series in order to obtain the electron temperature in the plasma. The measurements were made using the reduced 3-spark system. Two zones of the expanding hemispherical plasma were selected by appropriate diaphragms inside the gas cell. An outer zone was selected between 9 and $11 \mathrm{~mm}$ above the electrodes. An inner zone was selected between 4 and $6 \mathrm{~mm}$ above the eiectrodes. The intensity of the He (I) lines as properly corrected in accordance with the response of the whole optical system, lenses, monochromator, and photomuitiplier. The detection system response was determined using NBS calibrated tungsten-ribbon lamp.
According to Griem, ${ }^{12}$ a level $n$ is in local thermodynamic equilibrium (LTE) with level $n+1$ if the electronic density is over a value $n_{e}$, where $n_{e}$, in $\mathrm{cm}^{-3}$, is given by

$n_{e}=\left(\frac{7.4 \times 10^{8}}{n^{17 / 2}}\right)\left(\frac{k T_{e}}{13.6}\right)^{1 / 2} \exp \left(-\frac{\left(E_{n}-E_{n+1}\right)}{k T_{e}}\right)$,

where $E_{n}$, the energy of level $n$, and $T_{e}$ are in $\mathrm{eV}$.

Under the discharge conditions of these experiments, the electron density is measured to be in the vicinity of $1 \times 10^{12} \mathrm{~cm}^{-3}$, and the electronic temperature $T_{e} \geqslant 600 \mathrm{~K}$. At these conditions, levels with $n \geqslant 6$ will be in LTE within $10 \%$, with the neighboring level. In addition to the fulfilment of the criterian of Eq. (1), it is necessary ticat changes in electron temperature are small over time, $\tau_{n}$ characterizing the establishment of an equilibrium. For the levels of interest and the values of $n_{e}, T_{e}$ found in this work, result in $\tau_{n}<10^{-7} \mathrm{s.}^{12}$ This short time implies that the transient nature of the plasma does not cause deviation from LTE between excited-state populations in our measurements.

Figure 1 shows a partial spectrum of the light emitted by the reduced 3-spark device. The light was selected from the region centered $10 \mathrm{~mm}$ above the strips and the spectrum was taken with the Boxcar gated $50 \mu$ s after the current pulse. Four lines of the $2 s^{3} S-n p^{3} P$ He series with $n \geqslant 6$ can be observed.

If levels $n$ and $n^{\prime}$ are in LTE, the intensity of the lines originated there are related to $k T_{\mathrm{e}}$ by

$k T_{e}=-\left(E_{n}^{\prime}-E_{n}\right)\left[\ln \left(\lambda^{\prime 3} n^{\prime 3} I_{n^{\prime}} / \lambda^{3} n^{3} I_{n}\right)\right]^{-1}$

where $\lambda$ and $\lambda$ ' are the wavelengths of the lines with intensities $I_{n}$ and $I_{n^{\prime}}$, respectively.

Neutral density filters reduced the intensity of the strongest lines. $\mathrm{Cd}(\mathrm{I})$ and $\mathrm{Cd}$ (II)lines appearing in this spectral region are also shown. Figure 2 shows the results for $\ln (1 /$

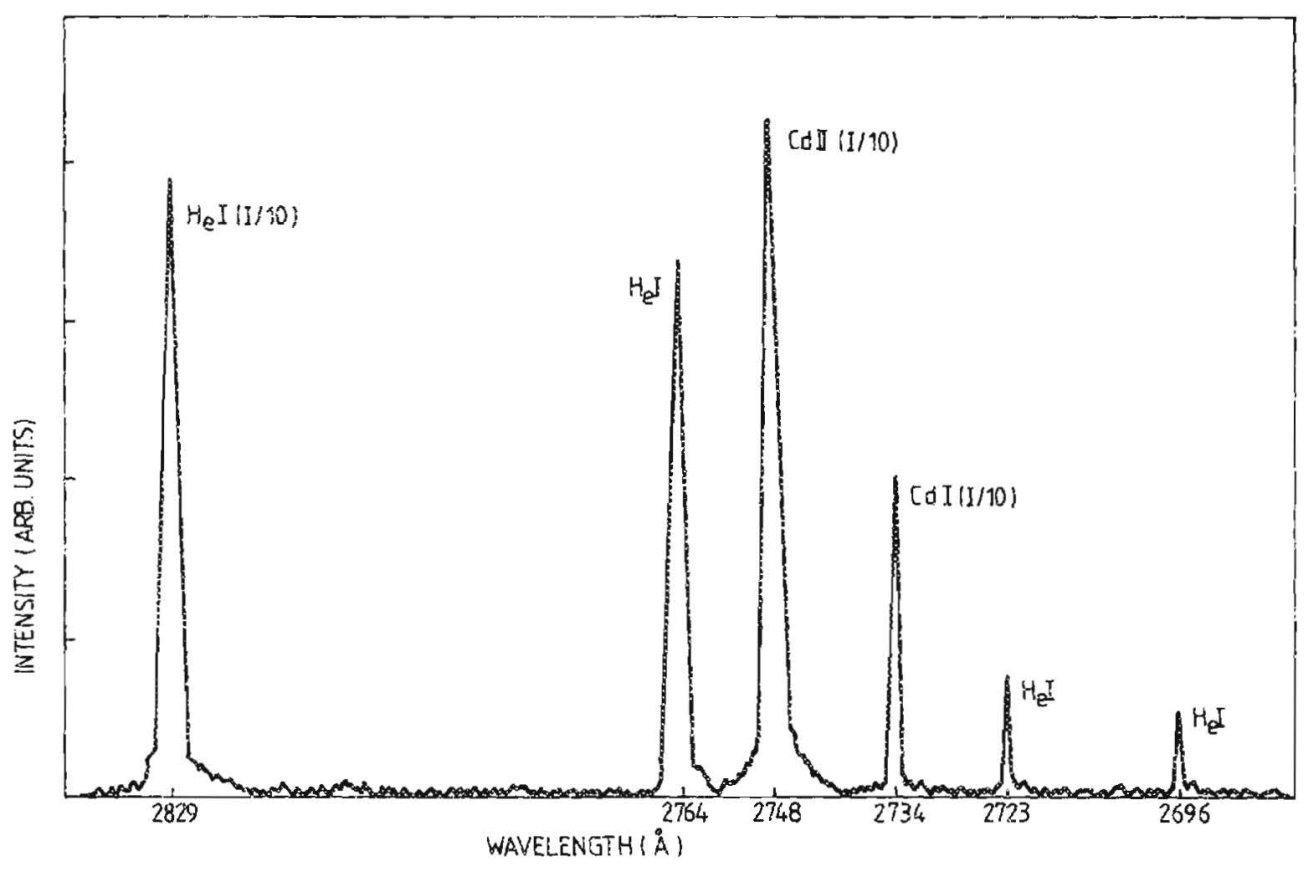

FIG. 1. The partial spectrum of the He-Cd spark discharge obtained from the plasma region $10 \mathrm{~mm}$ over the $\mathrm{Cd}$ strips. The intensity was recorded 50 $\mu$ after the current pulse. Some highintensity lines are attenuated by ten. 


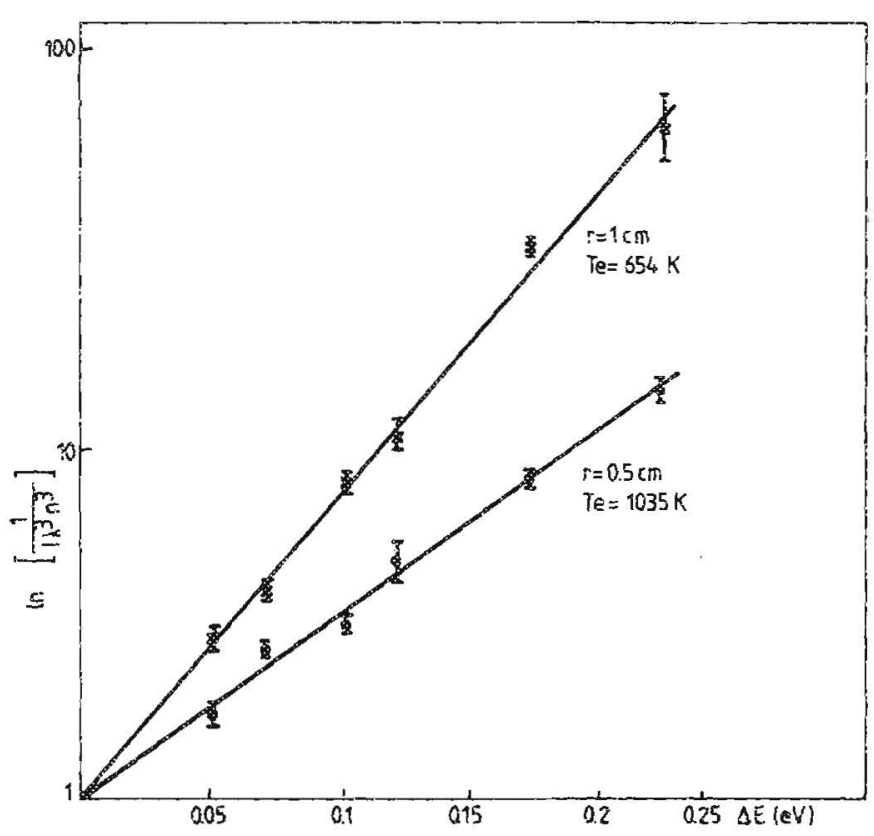

FIG. 2. The semilogarithmic plots of line intensities of the $2 s^{3} S-n p^{3} F$ He (I) series. The values were obtained $50 \mu \mathrm{s}$ after the discharge. The radial position and corresponding values for $T_{e}$ are indicated at the right.

$\left.\lambda^{3} n^{3} I\right)$ vs $\Delta E\left(n, n^{\prime}\right)$ for the light coming from regions centered 5 and $10 \mathrm{~mm}$ over the strips at $50 \mu \mathrm{s}$ after the discharge firing. Data ior each condition were fitted by straight lines using a least-squares routine. The values for $T_{e}$ given in the figure were calculated using Eq. (2). The relative errors were below $20 \%$ showing that LTE is reached for the levels involved. The values for $T_{e}$ were substantially lower than the values found in the axially excited $\mathrm{Sr}$ (II)-recombination iaser $^{3}$ and used for early calculations of electron density limits in plasma-recombination lasers. ${ }^{10}$

By the use of an electronic gate in different temporal positions we determined the electronic temperature over the time range $20-100 \mu$ s after the current pulse. Before $20 \mu \mathrm{s}$, the measurements were made all the more difficult by the noise related to the discharge current; after $100 \mu \mathrm{s}$, the intensities of $\mathrm{He}$ (I) lines were negligible. In Figure 3, we show the time deperdence of the intensity of $\mathrm{He}$ (1) lines used in the temperature measurement. Diaphragms were used to select the light coming from the region situated 9-11 mm over the strips. Figure 4 shows the electron temperature evolution for two plasma regions, situated at 5 and $10 \mathrm{~mm}$ from the strips. The temperature is relatively constant during the laser oscillation around $40 \mu \mathrm{s}$, but the cooling produced by the plasma expansion is clearly seen.

\section{Particle density measurements}

The ground-state densities of $\mathrm{Cd}$ (I) and $\mathrm{Cd}$ (II) were measured using the absorption technique of Landeuberg and Reiche. " The two-gap reduced device was usea in this part of the experiment. One of the spark gaps is used as a light source to measure the absorption by the second gap. Both sparks are identical by construction. Since they are operated in the same cell and are in series, the temperatures in both plasmas are the same. Consequently the linewidths in both

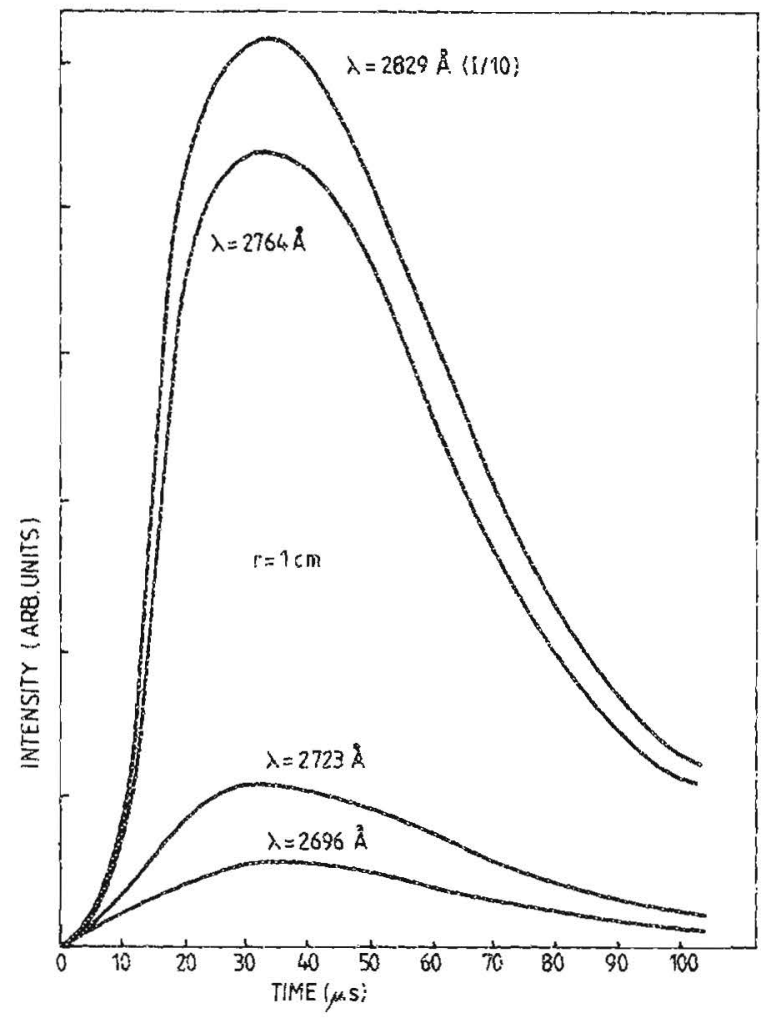

FIG. 3. The temporal profile of $\mathrm{He}$ (1) lines used to determine the elecironic temperature. The radial position is $10 \mathrm{~mm}$ over the strips.

sparks can be expected to be equal. The light coming from zones situated at 4-6 $\mathrm{mm}$ or 9-11 $\mathrm{mm}$ from the sparks was properly selected by the use of diaphragms. The density of $\mathrm{Cd}$ (I) and $\mathrm{Cd}$ (II) ground states were determined using the $3261,2288 \AA$ and $2144,2265 \AA$ lines, respectively. The absorption coefficients measured in 1-cm-long plasmas were $k_{0}(3261 \AA)=0.11+0.03 \mathrm{~cm}^{-1}$ and $k_{0}(2144$ $\AA)=0.93 \pm 0.05 \mathrm{~cm}^{-1}$ in the region closer to the gaps, and $k_{0}(2288 \AA)=0.11 \pm 0.02 \mathrm{~cm}^{-1}$ and $k_{0}$ (2265 $\AA)=2.92 \pm 0.04 \mathrm{~cm}^{-1}$ at $10 \mathrm{~mm}$ from the gaps. Using the

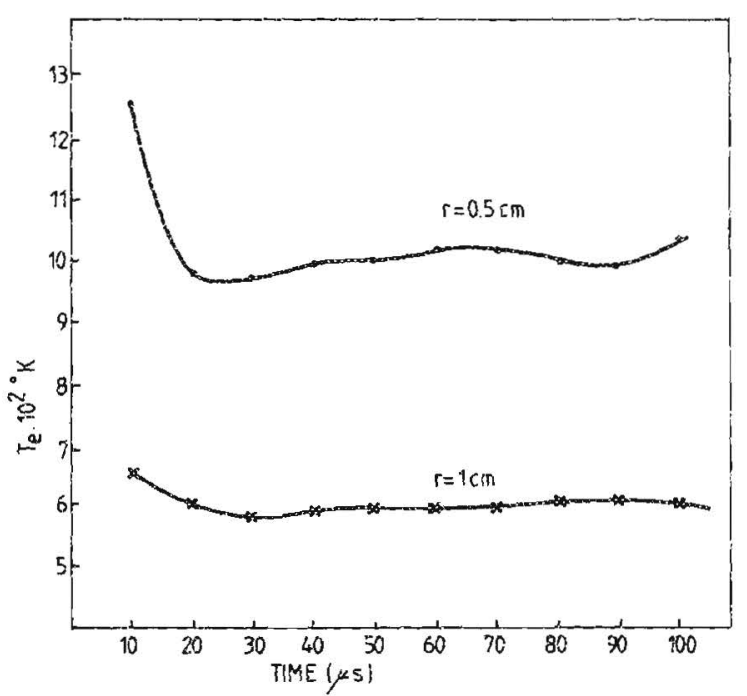

FIG. 4. The temporal dependence of the electronic temperature; the radial positions over the stips are shown. 
values for $k_{\theta}$, the densities for the $\mathrm{Cd}(\xi)$ and $\mathrm{Cd}(\mathrm{I})$ at $40 \mu$ s after the discharge pulses were found to be $[\mathrm{Cd}(\mathrm{I})]=(3 \pm 1) \times 10^{11} \mathrm{~cm}^{-3}$

[Cd (II)] $=(2.0 \pm 0.1) \times 10^{12} \mathrm{~cm}^{-3}$ at $5 \mathrm{~mm}$ over the strips, and $[\mathrm{Cd}(\mathrm{I})]=(6 \pm 1) \times 10^{9} \mathrm{~cm}^{-3},[\mathrm{Cd}(\mathrm{II})]=(9.8 \pm 0,1)$ $\times 10^{11} \mathrm{~cm}^{-3}$ at $10 \mathrm{~mm}$ from the strips. The ion densiry is in good agreement with the measurement of Wood et al. ${ }^{10}$

Knowledge of the plasma densiry is important to caiculate the recombination rate, collisional de-excitation rates, and optical gain. Emission from the most intense lines of $\mathrm{Cd}$ (III) (e.g., 2767, 2805, and $3035 \AA$ ) was undetectable at the time when laser action took place $(25-55 \mu \mathrm{s})$. Multiply charged ions, that might be created during the discharge recombine at eariy times in the afterglow. The positively charged particles at the time of laser oscillation are reduced to $\mathrm{Cd}$ (II) and He (II).

We have determined the $\mathrm{Cd}$ (II) density by absorption measurements as previousily described. Similar measurements of $\mathrm{He}$ (II) are made all the more difficult by the short wavelength of the $\mathrm{He}$ (II) resonant lines. However, a study of the intensity of spectral lines at the time significant optical gain is observed, show that the He (II) concentration can be neglected as compared with the Cd (II) density. We compared the intensities of $\mathrm{Cd}$ (I) lines excited by recombination of $\mathrm{Cd}$ (II) with the intensity of a large number of $\mathrm{Cd}$ (II) lines that originate from levels that are excited by change transfer reactions from $\mathrm{He}$ (II), and with He (I) lines excited by recombination of helium ions. During the time of the laser action, the $\mathrm{Cd}$ (II) and $\mathrm{He}(\mathrm{l})$ lines are more than one order of magnitude less than the lines of the Cd (I) spectrum. As an example, the time evolution of the intensity of three spectral lines corresponding to $\mathrm{Cd}$ (I), $\mathrm{Cd}(\mathrm{II})$, and $\mathrm{He}(\mathrm{I})$ is shown in Fig. 5. The evolution of the $1.433-\mu \mathrm{m}$ laser intensity is also shown to provide a time reference, its intensity being in a different scale. In summary, our observations confirm, as previously concluded, ${ }^{10}$ that the plasma density is practically equal to the $\mathrm{Cd}$ (II) density at the time when laser action occurs.

\section{Gain measurements}

The optical gain of the $1.433-\mu \mathrm{m}$ laser termination of Cd (II) was measured using the 33-strip laser. A rotating glass plate was installed inside the gas cell in order to introduce a calibrated loss by tilting it with respect to the Brewster angle. The coupling loss due to the hole in the output mirror was calculated to be $4 \%$. When the resonator was aligned $7 \mathrm{~nm}$ over the strips, the single pass gain was measured to be $9 \%$ for the $36-\mathrm{cm}-$ long active medium. This corresponds to a small signal gain coefficient of $2.5 \times 10^{-3}$ $\mathrm{cm}^{-1}$.

\section{OPTICAL GAIN CALCULATIONS}

We considered that the $\mathrm{Cd}$ (II) ions recombine by a three-body collisional process to populate the tigh-energy levels of $\mathrm{Cd}(\mathrm{l})$. By collisional and radiative cascade, these levels are assumed to populate the upper level of the 1.433$\mu \mathrm{m}$ line at a rate $P$, given by

$$
P=\alpha \mathrm{Cd}(\mathrm{I}) n_{e}^{2} T_{e}^{-9 / 2},
$$

where $\alpha$ is the three-body collisional recombination coeficient. The upper- and lower-laser levels are depleted by radiative decay and superelastic electron de-excitation. The lower level was assumed to be populated by radiative and collisional decay from the upper level. Published $\mathrm{Cd}$ (I) radiative lifetimes ${ }^{13,14}$ and electron de-excitation rates derived by Wood et al. ${ }^{10}$ were used.

Assuming that the plasma density is equal to the concentration of $\mathrm{Cd}$ (II) ground-state ions, and using the measured values: $T_{e}=600 \mathrm{~K}$ and $\mathrm{Cd}(\mathrm{II})=9.8 \times 10^{1 \mathrm{i}} \mathrm{cm}^{-3}$ at

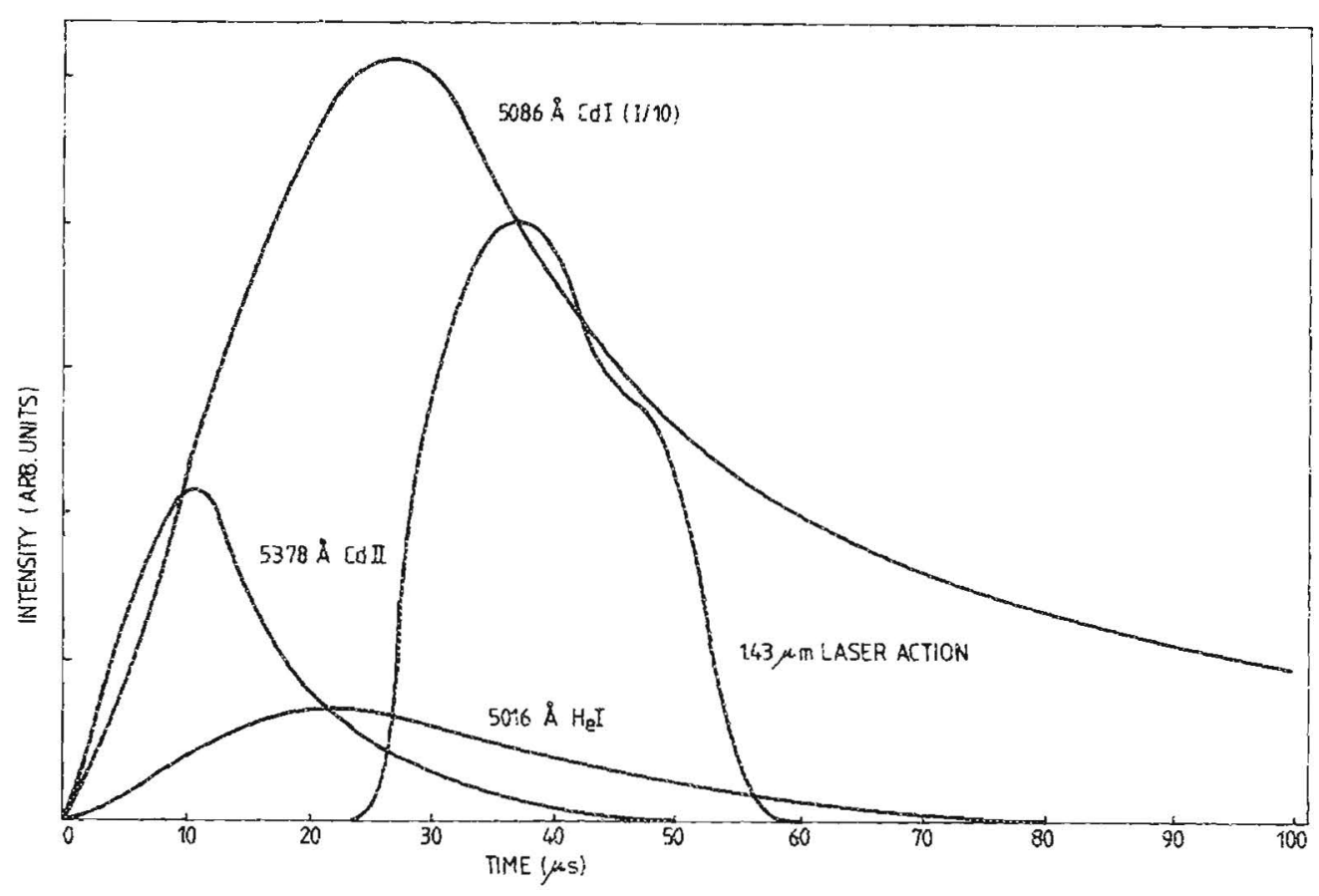

FIG. 5. The intensity of lines related to $\mathrm{Cd}$ (II) density and $\mathrm{He}$ (I) derisity, as a function of time after the discharging firing. The $\mathrm{Cd}(\mathrm{I})$ line intensify is reduced by ten. The evoIntion of the laser output in the $1.433-\mu \mathrm{m} \mathrm{Cl}(\mathrm{I})$ line is also shown; its intensity is in a different scale. 
$10 \mathrm{~mm}$ from the gaps, a small signal gain of $3 \times 10^{-3} \mathrm{~cm}^{-1}$ is obtained. The calculated gain in the region at $5 \mathrm{~mm}$ from the sparks, measured at $T_{\mathrm{e}}=1000 \mathrm{~K}$ and $\mathrm{Cd}$ (II) $=2 \times 10^{12}$ $\mathrm{cm}^{-3}$, was $1.5 \times 10^{-3} \mathrm{~cm}^{-1}$. The value of the gain measured aligning the resonator axis at $7 \mathrm{~mm}$ from the gaps, $2.5 \times 10^{-3}$ $\mathrm{cm}^{-1}$, falls in between the calculated values at 5 and $10 \mathrm{~mm}$.

\section{SUMRMARY}

We have made space- and time-resolved measurements of the electron temperature in the plasma of a cadmium SPER-recombination laser. The electron temperature at 10 $\mathrm{mm}$ from the discharge gaps, was found to be $600 \mathrm{~K}$ at the time of maximum gain. At the same time $T_{e}$ at $5 \mathrm{~mm}$ from the gaps was found to be $1000 \mathrm{~K}$.

Most of the plasma cooling oceurs in the first several $\mu$ s after the termination of the current pulse when both the plasma expansion and elastic collisions make their maximum contribution to the cooling of the electron gas. At this time, the rate of change of the plasma volume and the difference between $T_{e}$ and the atomic and ionic temperatures are maximum. After this initially rapid decay, the rate of change in $T_{e}$ is small, and $T_{e}$ at $10 \mathrm{~mm}$ from the discharge gaps remains at approximately $600 \mathrm{~K}$ for $100 \mu \mathrm{s}$. The rapid cooling of $T_{c}$ to thermal values is one of the fundamental reasons of the successful use of SPER devices in the generation of a significant number of new laser lines. ${ }^{15}$

The cadmium ground-state ion density at the condition of maximum gain was measured to be $9.8 \times 10^{21} \mathrm{~cm}^{-3}$ and $2 \times 10^{12} \mathrm{~cm}^{-3}$ in the region centered at 5 and $10 \mathrm{~mm}$ from the sparks, respectively. No evidence of significant concentrations of other ion species was found at the time laser action took place, confirming that the plasma density is practically equal to the density of $\mathrm{Cd}(\mathrm{II})$, as previousiy suggested. ${ }^{10}$
The magnitude of the small signal gain of the $\mathrm{Cd}(\mathrm{l})$ $1.433-i \mathrm{~m}$ line calculated using the measured values of the plasma parameters is in good agreement with the measured gain of $2.5 \times 10^{-3} \mathrm{~cm}^{-1}$.

\section{ACKHOWLEDGMENTS}

This work was supported by CONICET (PID $3.922907 / 85$ ) and CIC. J. Rocca wants to acknowledge the support of an N.S.F. Presidential Young Investigators Award N. S. International Grant INT-8402914 and NSFGrant ECS-8606226. Fruitful discussions with Dr. H. F. Ranea Sandoval are acknowledged.

'L. 1. Gudzenko and 1. A. Sheiepin, Zh. Eksp. Teor. Fiz. 45, i445 (1963) [Sov. Phys. JETP 18, 998 (\$964)].

${ }^{2}$ L. I. Gudzenko, L. A. Shelepin, and S. I. Yakovelenko, Sov. Phys. Usp. 1\%, 848 (1975).

${ }^{3}$ V. V. Zhukov, V.S. Kucherov, E. L. Latush, and M. F. Sém, Sov. J. Quantum Electron. 7, 708 ( 1977 ).

${ }^{4}$ E. M. Campbeil, R. G. Jahn, W. F. von Jaskowsky, and X. E. Clark, J. Appl. Phys. 51, 109 (1980).

'M. Brandi, Appl. Phys. Lett. 42, 127 (1983).

6y. J. Rocca, Appl. Phys. Lett. 47, 1145 (1985).

7O. R. Wood, II, J. J. Macklin, and W. T. Silfvast, Appl. Phys. Lett. 44, 1123 (1984); and IEEE J. Quantum Electron. QE-21, \{714 (1985).

${ }^{8}$ W. T. Silfvast, L. H. Szeto, and O. R. Wood, II, Appl. Phys. Iett. 36, 615 (1980).

${ }^{9}$ E. Hinnov and J. G. Hirschberg. Phys. Rev. 125, 795 (1962).

10 O. R. Wood, II, and W. T. Silfvast, Appl. Phys. Lett. 41, 121 (1982).

"A. C. G. Mitchenl and M. W. Zemansky, Resonance Radiation and Excited Atoms (Cambridge University, New York, 1961).

${ }^{12}$ H. R. Griem, Phys. Rev. 131, 1170 (1963).

${ }^{13}$ Ya. F. Veralinen and V. I. Irivalov, Opt. Specirosc. 42, 131 (1979).

${ }^{14}$ V. A. Zilitis, Opt. Spectrasc. 31, 86 (1971).

is S. T. Silfvast, L. H. Szeto, and O. R. Wood, Appl. Phys. Lett. 39, 212 (1981). 